\title{
El eliminativismo filosófico y su ataque a la psicología*
}

\author{
Ricardo A. Braun
}

En este artículo se discuten las tesis contemporáneas del eliminativismo, forma radical de reduccionismo de los términos mentalistas y explicaciones causales usadas en psicología, que presuponen los conceptos de creencias y deseos. Si bien el uso de las categorías de creencias y deseos en la explicación de la conducta humana es característico de la llamada "psicología popular", la psicología científica también hace uso de estas categorías y, por lo tanto, la tesis eliminitavista amenaza en último término a la misma psicología científica. Se presentan las tesis de P. M. Churchland, de P. S. Churchlandy de S. Stich, argumentando que el programa eliminativista es inadecuado por las siguientes razones: 1) la particular naturaleza de la explicación psicológica; 2) nuestro autoconcepto como seres racionales; y (3) factores pragmáticos que hacen inviable tal proyecto.

eliminativismo / reduccionismo / psicología de creencias y deseos / explicación científica

\section{Eliminativism in philosophand the attack to scientific psychology}

In this paper the current theories of eliminativism are discussed. Eliminativism is the radical reductionist program of mental terms and causal explanation as it is used in psychology which presupposes the concepts of beliefs and desires. Although the use of beliefs and desires is commonplace in the so-called "Folk psychology", ultimately scientific psychology utilizes those categories as well, and therefore, eliminativism is also an attack to scientific psychology. The theses of $P$. M. Churchland, P. S. Churchland and S. Stich are discussed and I shall demonstrate that eliminativism is an inadequate program due to: 1) the unique nature of pschological explanations; 2) our self-notion as rational beings; and 3) pragmatic concerns.

eliminativism / reductionism / belief-desire psychology / scientific explanation

* Este artículo es el resultado parcial de la investigación "Psicología popular y psicología científica" auspiciada por el Instituto de Investigación Científica de la Universidad de Lima. Agradezco al Instituto por el apoyo para la realización de este estudio y por la autorización para publicar parte del trabajo. 


\section{INTRODUCCIÓN}

El eliminativismo no es una tesis usualmente discutida en la psicología, ya que tanto la terminología como los autores más representativos pertenecen al quehacer filosófico. Sin embargo, las propuestas eliminativistas como programa radical reduccionista tienen serias implicancias en la fundamentación de la psicología científica, que basa su modelo explicativo en una manera análoga a la empleada por la psicología popular.

El eliminativismo, como el nombre sugiere, propone no realizar reducciones progresivas y por pasos de los fenómenos mentalistas denominados "creencias" y "deseos", o un eventual reemplazo conceptual de estos, sino más bien la eliminación radical de todo nuestro aparato explicativo basado en esos fenómenos y en las relaciones causales entre las creencias y deseos que postula, para dar paso, en su lugar, a una neurología madura en forma universal.

\section{HISTORIA DEL CONCEPTO}

Desde hace dos décadas, aproximadamente, la tesis del eliminativismo ha tenido un protagonismo en las discusiones de la filosofía de la mente y la filosofía de la psicología. La tesis también ha sido llamada eliminativismo materialista. La doctrina materialista es más bien antigua en la tradición filosófica, si pensamos en Demócrito y luego en
Hobbes, que propugnaron una visión fisicalista, y, por ende, naturalista del ser humano. El ser humano de Demócrito, de Hobbes y de otros materialistas, tendría una sola base substancial física o material. El eliminativismo también podría atribuirse a la posición de Hume acerca de nuestra concepción ordinaria del Yo, descartándola como una sustancia independiente. Por ello, conceptos como alma y espíritu quedarían descartados en la economía ontológica. Sin embargo, el término eliminativismo materialista, o simplemente eliminativismo, es producto del siglo XX y está específicamente dirigido contra la existencia de estados mentales que asociamos con creencias y deseos.

El eliminativismo del siglo XX tiene cuatro figuras relevantes en su inicial formulación: W. Sellars, W. V. O. Quine, P. Feyerabend y R. Rorty. El panorama en la filosofía de la mente a mediados del siglo XX estaba dominado por la teoría de la identidad mentecerebro, pero ciertamente había claros remanentes de algunas convicciones emanadas del Círculo de Viena, particularmente la visión acerca del lenguaje ordinario y su puesto en la ciencia. Así, para Quine, todos los términos asociados al de las creencias deberían ser eliminados por otros que tuvieran un claro correlato fisiológico.

En Palabra y objeto, Quine considera que los términos que denotan los correlatos físicos de los estados mentales serán más útiles que sus contrapar- 
tes mentalistas, y afirma: "Si los estados corporales existen de todos modos, ¿para qué añadir otros más?” (1960, p. 264). Es importante mencionar que Quine no encuentra una diferencia notable entre explicar los estados mentales como estados fisiológicos - clara influencia de la teoría de la identidad de Place (1956) y Smart (1959) - y eliminar los términos mentales a favor de los términos de estados físicos.

Se pregunta en la misma obra: “... ¿repudia [la teoría fisicalista] el estado mental de dolor o cólera a favor de su concomitante físico? ¿O identifica el estado mental con el estado del organismo físico?" (1960, p. 265). Quine considera que no hay mayor diferencia: "Algunos encontrarán consuelo reflexionando que la distinción entre un fisicalismo eliminativo y explicativo es irreal" (p. 265).

Por su parte, Feyerabend sostuvo que con el tiempo se probaría que la psicología de sentido común es radicalmente falsa. Para Feyerabend toda forma de materialismo es una forma de eliminativismo - de ahí la relación casi necesaria de los términos- de la psicología popular. La tensión que advierte Feyerabend es metafísica: los conceptos de la psicología popular son de naturaleza no físicos, por lo tanto, cualquier forma de fisicalismo o materialismo implicaría necesariamente que no existen procesos mentales o estados tales como los entiende el sentido común (Feyerabend, 1963).
Podemos ver que en los primeros eliminativistas son dos las posiciones acerca de la psicología popular:

1) Los conceptos mentales son equivalentes a conceptos vacíos, puesto que sus términos se refieren a algo que no existe.

2) Los estados mentales existen, pero son simplemente estados cerebrales.

El eliminativismo de acuerdo a 1) es un eco de la eliminación de muchos conceptos, con sus respectivos términos, que resultaron hacer referencia a cosas inexistentes, como, por ejemplo, los demonios, las esferas de cristal y conceptos parecidos. De acuerdo a 2), hay un reconocimiento de los estados mentales -en oposición al conductismo lógico y metodológico original- pero requiere una reconceptualización teniendo como marco conceptual a las neurociencias. Es decir, no es que los estados mentales no existan, sino que están inadecuadamente descritos en el lenguaje popular mentalista y debieran ser traducidos o reemplazados por conceptos neurológicos en una futura ciencia de la conducta.

Las dos posiciones provocaron dudas acerca del programa mismo del materialismo eliminativista, especialmente la segunda, puesto que se asemejaba mucho al materialismo reduccionista. En todo caso, para hacer las cosas más confusas aparece el trabajo de Rorty (1965), que endosa ambas posturas asociadas al eliminativismo. 
En efecto, para Rorty las sensaciones y categorías semejantes eran inexistentes; y además, lo único que existía eran los procesos cerebrales. Pero el problema de Rorty es que, en esta situación, no se pueden tener ambas posturas. Por un lado, si se niegan los acontecimientos mentales, y en ese caso se convierte en materialista eliminativista radical, no tiene lugar la afirmación de que lo único que hay son eventos cerebrales, puesto que estos eventos serían lo que la tradición mentalista ha venido llamando eventos mentales, con lo cual su posición sería la de un reduccionista ortodoxo. Pero en todo caso, las posiciones son incompatibles entre sí, y por lo tanto Rorty tenía que haber escogido entre una de las dos.

En nuestros días, el materialismo eliminativista ha provocado nuevamente la atención de filósofos, psicólogos y científicos cognitivos, especialmente por las implicancias en el permanente problema de la metafísica de lo mental, el proceso de cambio de las teorías, la naturaleza de la explicación psicológica y los recientes desarrollos en la ciencia cognitiva. Los argumentos eliminativistas están generalmente asociados, por un lado, a los trabajos de Paul Churchland y Patricia Churchland, y por el otro, a los de Stephen Stich. Las posiciones no son iguales en cuanto al contenido y proceso eliminativista entre estos filósofos. Como veremos con más detalle a continuación, los Churchland abogan radicalmente por la eliminación de todo el vocabulario y la ontología que postula la psicología popular. Stich, más moderado, reconoce que si bien es cierto, la psicología computacional convencional de alguna manera ha reivindicado a la psicología popular, sin embargo, en un futuro, las taxonomías que corresponden a la psicología popular debieran ser rechazadas plenamente.

\section{EL ELIMINATIVISMO DE LOS \\ CHURCHLAND}

Independientemente de las posturas que se tengan, es innegable el aporte de Paul y Patricia Churchland al debate acerca del eliminativismo. La prueba están en el constante debate a favor y en contra de ellos que caracteriza a la filosofía analítica de la mente en los últimos veinte años. Los Churchland han cuestionado muchas de las nociones mantenidas tradicionalmente en áreas de la epistemología, la ciencia, el lenguaje y la mente.

Los Churchland tienen un punto de ventaja con respecto a los filósofos tradicionales: sus teorías han sido puestas a prueba dentro del campo empírico de la ciencia y con ello han conseguido una relación entre la filosofía de la mente y la neurociencia. De esta investigación han resultado nuevas disciplinas, acuñadas como "programas neurofilosóficos" y "programas neurocomputacionales". Por ejemplo, recientemente Paul Churchland ha abordado el 
complejo tema del conocimiento moral desde una perspectiva neurocomputacional en su The engine of reason, the seat of the soul (1995).

La neurofilosofía y el neurocomputacionalismo retoman los trabajos de la epistemología naturalizada de Quine, la visión revolucionaria de la ciencia de Kuhn, el ataque de Sellars al "Mito de lo dado" y la propuesta eliminativista radical de Feyerabend.

Los Churchland han mantenido un ataque constante contra la psicología popular sosteniendo que esta ofrece un terriblemente inadecuado programa para la investigación, explicación y predicción del comportamiento humano y la vida mental. Por ello, vislumbran una eventual eliminación tanto del lenguaje como de la ontología acompañante de la psicología popular en vista de los progresos de las neurociencias.

Los argumentos, como veremos, están orientados a demostrar que la psicología popular padece males incurables, entre ellos la incapacidad de explicar una serie de fenómenos psicológicos, un estancamiento teórico y empírico a través de los siglos, y su discontinuidad con otras disciplinas que abordan el problema de la conducta humana, y, en general, un fracaso del uso de la psicología popular en otros campos empíricos.

Como ya se mencionó, el desafío que siempre provocan los Churchland es que ante la catastrófica prognosis que elaboran para la psicología popular, aparecen con modelos alternativos que siempre están científicamente informados y en áreas tradicionalmente asignadas a la filosofía y la psicología mentalista: percepción visual, integración sensorio-motriz, aprendizaje, memoria, cualidades sensoriales y comprensión. Sin embargo, dado que el trabajo de los Churchland, si bien es cierto ha sido publicado como autores separados, sus convicciones tienen mucho en común, y para los efectos de este artículo los presentaré como unitarios, alternando las fuentes de uno y otro como ilustración.

Primero, veamos la concepción que tienen sobre el materialismo eliminativista. Se puede resumir en la siguiente cita de P. Churchland:

Es la tesis que nuestra concepción común de los fenómenos psicológicos constituye una teoría radicalmente falsa, una teoría tan fundamentalmente defectuosa que tanto los principios y la ontología de tal teoría eventualmente serán desplazadas, en vez de suavemente reducidas, por una neurociencia completa. Nuestra comprensión común y aun nuestra introspección será entonces reconstituida dentro del marco conceptual de una neurociencia completa, una teoría que podemos esperar sea mucho más poderosa que la psicología de sentido común que desplaza, y sustancialmente más integrada dentro de la ciencia física en general (1991, p. 601).

En segundo lugar, los Churchland, como se aprecia en la cita, parten de la aseveración de que la psicología popu- 
lar es una teoría, en consecuencia, su estrategia será de enfrentarla como una teoría empírica al modo que estudiamos, analizamos y juzgamos una teoría científica. Pero, ¿por qué una teoría? Según Churchland, la psicología popular es un marco conceptual que unifica la mayoría de los fenómenos que han preocupado a la filosofía de la mente, entre ellos, la explicación y predicción del comportamiento, la semántica de los predicados mentales, la teoría de la acción, el problema de las otras mentes, la intencionalidad de los estados mentales, la naturaleza de la introspección y el problema mente-cuerpo. Un esfuerzo por reunir todos estos temas y problemas dentro de un marco conceptual, merece ser tomado seriamente por cualquiera que los estudie. En consecuencia, dada la unificación que hace la psicología popular acerca de diferentes y complejos problemas de la vida mental humana, merece ser considerada una teoría entendida como un marco conceptual que integra y le da funcionalidad. Toda persona que utiliza la psicología popular se está apropiando del vocabulario, generalizaciones y marco conceptual de esta teoría, de forma análoga a cómo lo hacemos con otras teorías científicas. Sin una teoría, para Churchland, las personas no seríamos capaces de explicar y predecir el comportamiento de otras personas con la facilidad y éxito que son dignos de destacar (1981/1991, p. 602).
En tercer lugar, las actitudes proposicionales forman el núcleo de la psicología popular y su aparente ininteligibilidad como productos de estados mentales intencionales no debiera emerger como un misterio sino como una característica estructural de los conceptos de la psicología popular. Curiosamente, los Churchland vinculan las características estructurales de la teoría de la psicología popular con las teorías de las ciencias físicas (p. 602).

Finalmente, los Churchland se preguntan: ¿cómo es posible que se relacionen las entidades de una teoría (psicología popular) con la ontología de otra teoría (neurociencia completa)? Pero, como ya se dijo, el veredicto es claro. No hay posibilidades de supervivencia de la teoría de la psicología popular ante una eventual reducción, puesto que es o muy confusa o muy defectuosa para sobrevivir una reducción interteorética. El único camino que queda es el desplazamiento teórico, por ser una teoría estancada (Lakatos, 1970).

Los argumentos que sostienen el veredicto son conocidos, pero es importante mencionarlos con miras a su posterior análisis. Primero, destacan el problema de la capacidad explicativa de la psicología popular, o más bien de su incapacidad explicativa. Dentro de la teoría de la psicología popular no hay lugar para la explicación de la enfermedad mental, la facultad de la imaginación creativa, ni la fuente de las di- 
ferencias de inteligencia entre los individuos. A estos casos se puede añadir la ignorancia popular acerca de la naturaleza y funciones psicológicas del sueño. Peor suerte corren las explicaciones de cogniciones más complejas como la construcción interna de una imagen en 3-D a partir de un conjunto de estimulaciones en 2-D en nuestras retinas. $\mathrm{O}$ también nos piden que pensemos en las características de la memoria, las ilusiones perceptivas, visuales o de otro tipo. A todo esto, la psicología popular se mantiene en un ignorante silencio.

Con una mirada condescendiente, los Churchland no concluyen que la psicología popular es una teoría falsa para la mayoría de las personas, pero sostienen que, en el mejor de los casos, es tan superficial, parcial e incapaz de iluminar que, al final, es inadecuada para formar un sistema que permita comprender mejor la compleja realidad. Por ello, la teoría está condenada a desaparecer en el campo de la ciencia. Los Churchland no se animan a sostener que la psicología popular es radicalmente falsa como una guía para la vida práctica. Lo que quieren decir cuando juzgan a la psicología popular como fuente de la psicología científica es que está equivocada con respecto a los estados internos que subyacen a nuestro comportamiento. Específicamente, es equivocado suponer que tenemos pensamientos, creencias, deseos, esperanzas y otros estados intencionales como cosas reales.
El estancamiento de la teoría de la psicología popular es una prueba más de la infertilidad y decadencia que anuncian las características de las seudociencias (Thagard, 1978). Se puede comparar la psicología popular de los griegos con la de hoy día y veríamos que es, sin sorpresas, muy similar. Las explicaciones y predicciones actuales no son en nada mejores a las que podría recurrir Sófocles con su psicología popular. Claro, los Churchland no quieren ser injustos con la psicología popular y consideran que hay teorías que requieren tiempo para madurar. Pero eso sería cierto si no hubiera una teoría alternativa o si se pudiera esperar para reducirla eventualmente en otra teoría más competente. Sin embargo, para la psicología popular ya existe la alternativa, y esa es la neurociencia que promete desplazar y no integrar. En este sentido, dice P. Churchland:

La psicología popular no es parte de esta creciente síntesis. Sus categorías intencionales se erigen magníficamente solitarias, sin ninguna perspectiva de reducción en un corpus más extenso... La impotencia explicativa de la psicología popular y su largo estancamiento nos inspira poca fe en que sus categorías podrán encontrarse claramente reflejadas en el marco conceptual de la neurociencia. Al contrario, se nos recuerda cómo la alquimia debe haber parecido con el comienzo de la química elemental, cómo la cosmología aristotélica cuando empezaba a articularse la mecánica clásica, o cómo la concepción vitalista de la vida cuando tomaba cuerpo la química orgánica (1991, p. 604). 
La alternativa neurológica fue propuesta desde el inicio tanto en los trabajos de Paul como en los de Patricia. En efecto, en Consciousness: the transmutation of a concept (1983), Patricia S. Churchland rompe con la tradición de la interpretación del fenómeno consciente desde una perspectiva de sentido común y muestra una serie de descubrimientos clínicos y experimentales acerca de la conciencia. Su objetivo no era eliminar los fenómenos conscientes, sino aumentar el conocimiento, fecundidad, poder explicativo y coherencia de estos fenómenos con evidencia científica de tal modo que pudiera iluminar mucho más la discusión filosófica.

En el terreno metodológico, los Churchland han mantenido una motivación reduccionista, siguiendo la tradicional tesis de Nagel (1961). La tesis de Nagel se basa en el tipo de reducción denominada "interteorética". De acuerdo con esta tesis una teoría se reduce a otra si el vocabulario que hace referencia a sus entidades y propiedades son definibles, y sus leyes lógicamente derivables de la otra ("leyespuente"), conectadas por las identificaciones empíricas, correlaciones o definiciones reconstructivas. Schaffner (1967) complementó la versión de Nagel para permitir que hubiera aproximaciones y "sólidas analogías" en la conexión entre teorías. Shaffner propuso esta extensión del reduccionismo de Nagel para abarcar situaciones donde las teorías no coincidían exactamente después de la supuesta reducción, concordando con algunas predicciones, pero divergiendo de otras. La tradición de Nagel-Schaffner resultó en lo que hoy llamamos "reduccionismo internivel". En este tipo de reduccionismo se distinguen niveles de organización, y la explicación de los niveles más altos es realizada utilizando los niveles más bajos. Por ejemplo, en la genética clásica, antes de la era molecular, el genoma era representado jerárquicamente desde un alelo individual a múltiples alelos en un locus individual, luego a grupos de linaje, y finalmente a un genotipo multilocus entero. Las propiedades de un genotipo multilocus entero, esto es, todo el organismo, podía ser totalmente reducido - explicado- por los niveles más bajos de la jerarquía.

Los Churchland, al incorporar este modelo, han venido propugnando la reducción de la psicología en neurociencia, adaptando el modelo para lograr, según ellos mismos, un continuum de conmensurabilidad teórica en el sentido kuhniano (Kuhn, 1970). Por ello, su modo de reducción se asemeja más a la propuesta de Shaffner, pues sostienen que puede haber casos intermedios en los que mejor convenga una reducción aproximada.

En Neurophilosophy (1986), P.S. Churchland presenta lo que denomina la "co-evolución de las teorías" y que podría ser entendido como la inclusión de un elemento dinámico dentro del 
modelo de continuum. Su modelo coevolucionario permite no solo dinamismo en el tiempo en la relación entre dos teorías, sino que también introduce los medios para un análisis de muchas consideraciones que están implicadas en ese movimiento, a saber, el diseño del experimento, la técnica, los aparatos, las fuentes de evidencia y los recursos teoréticos y conceptuales.

Los Churchland sostienen que hay diferentes casos de relaciones interteoréticas que varían con respecto a la conmesurabilidad involucrada en las teorías. Para ello, proponen que tales casos sean contemplados dentro de un continuum de conmensurabilidad interteorética relativa. La inconmensurabilidad empieza a dominar cuando la ciencia revolucionaria toma lugar y se elimina la teoría inferior (Churchland \& Churchland, 1990).

\section{El eliminativismo de S. Stich}

A diferencia de los Churchland, que abogan por una eliminación inmediata de la psicología popular en el ámbito de la ciencia, Stich duda acerca de las categorías con las que se taxonomizan en la psicología popular, por lo tanto, niega que la psicología popular sea una teoría en absoluto, y que tenga lugar alguno en la futura ciencia psicológica (1983). En efecto, para Stich, lo que la psicología cognitiva computacional demuestra es que la manera como se taxonomiza los acontecimientos mentales no tiene nada en común con la forma como la psicología popular taxonomiza las actitudes proposicionales, lo que hace en forma de estados internos, causalmente relevantes. Por ello, para Stich resultaría que no hay tales estados como las actitudes proposicionales.

Algunas teorías científicas postulan la existencia de entidades que escapan a nuestra observación y descripción cotidiana y popular, como sucede por ejemplo en la física cuando sostiene la existencia de moléculas, campos y partículas subatómicas. Sin embargo, hay otras disciplinas, como la geología, que utilizan entidades que en gran medida proceden de los términos de los objetos comunes y macroscópicos. De forma análoga, algunos teóricos suponen que la psicología científica seguirá el camino de la geología, postulando las mismas entidades que la psicología popular, es decir, los estados intencionales en los términos en que se basan las explicaciones cotidianas del comportamiento, y con descripciones que apelan a los contenidos de tales estados de forma análoga a la psicología popular.

Para Stich, este camino es inviable. En la apuesta por el futuro de la psicología científica, Stich espera que eventualmente aprendamos acerca de los verdaderos procesos que subyacen a la cognición y se demostrará que las categorías que usa la psicología popular, en particular la categoría "creencia", no puede ser empíricamente defendida. Stich parte de la convicción de que para que los estados psicológicos sean 
relevantes en las explicaciones psicológicas, los organismos físicamente idénticos deben estar en los mismos estados psicológicos. Esta es llamada la "tesis de la superveniencia", que debemos, entre otros, a Kim (1993). Según él:

A superviene fuertemente sobre B solo en caso que, necesariamente, por cada " $x$ " y cada propiedad F en A, si " $x$ " tiene $F$, entonces hay una propiedad $G$ en $B$ tal que " $x$ " tiene $G$, entonces necesariamente si cualquier "y" tiene $\mathrm{G}$, entonces tiene F (1993, p. 65).

Aplicado a la teoría de lo mental en forma más simplificada nos quedaría lo siguiente:

Lo mental superviene en lo físico en que dos cosas cualquiera (objetos, eventos, organismos, personas, etcétera) exactamente iguales en todas sus propiedades físicas, no pueden diferir con respecto a las propiedades mentales. Esto es, la indiscernibilidad física implica necesariamente indiscernibilidad psicológica (Kim, 1992, p. 10).

Aparentemente, el argumento que sostendría Stich sería de defensa de un lenguaje materialista o fisicalista para la psicología, dada su aceptación del principio de superveniencia. Sin embargo, Stich está motivado por las doctrinas funcionalistas que, de alguna manera, sostienen una autonomía de lo psicológico frente al arreglo material físico en el que la función se realiza -y de ahí su interés por la ciencia cognitiva-.

Volvamos al problema de los organismos idénticos. Stich quiere demos- trar que, aun cuando dos organismos idénticos comparten la misma creencia, y si su historia externa es diferente, entonces, la individuación de la creencia sin tomar en cuenta la relación de verdad con el ambiente, -que es la forma como se clasifican las creencias en la psicología popular- resultaría en creencias que, si bien son distintas en sentido de su valor de verdad, resultan ser iguales para los dos organismos. $\mathrm{Si}$, además, se predica la eficacia causal de las creencias, Stich preguntaría ¿cómo sería posible que dos creencias que son diferentes con respecto al ambiente, sean iguales en la mente de los organismos que la comparten? Esta sospecha acerca de la falsedad de la tesis de la psicología popular que titula "tesis de la creencia-deseo" constituye la más clara evidencia de la inconveniencia de emplear el lenguaje de la psicología popular en la científica. Un ejemplo del mismo Stich aclarará su argumento:

Supongamos que tenemos la tecnología para crear réplicas de personas, átomo por átomo. Supongamos, además, que se acaba de crear una réplica mía. Yo creo que he probado una botella de Chateau d'Yquem, 1962. Si usted me preguntara si alguna vez he probado un d'Yquem '62, seguramente diría: "Si, lo he probado". El defensor de la tesis creencia-deseo aseguraría, con suficiente evidencia, que mi creencia está entre las causas de mi respuesta. Ahora, si se preguntara a mi réplica si alguna vez ha probado un d'Yquem, 1962, podría haber contestado posiblemente como "Sí, lo he probado". Y seguramente el teórico de la creencia-deseo consideraría que la creencia de mi réplica está entre las 
causas de su afirmación. Pero la creencia que es la causa de la afirmación de mi réplica tiene que ser de diferente tipo que aquella que es la causa de mi respuesta. Puesto que su creencia es falsa; acaba de ser creado y nunca ha probado un d'Yquem, ni ningún vino. De tal manera que... la propiedad de la creencia que él tiene como instancia es diferente a la mía (1991, p. 594).

Pero, como son réplicas, de acuerdo con el principio de autoría, debieran compartir todas las propiedades psicológicas explanatorias. Pero eso no es así, porque la propiedad de mi creencia de que he probado ese vino no es la misma que la propiedad de mi réplica. Y esa disimilitud es la que provoca la sospecha de Stich acerca de la individuación que hace la psicología popular. La creencia que tengo acerca de mí mismo es diferente lógicamente de la que tiene mi réplica, $y$, en consecuencia, según Stich, no puede tener lugar en una explicación científica de nuestro comportamiento.

Las creencias acerca de algo involucran relaciones con los objetos externos que las réplicas pueden no compartir. Si las réplicas físicas estuvieran localizadas en diferentes tiempos y lugares, sus creencias acerca del tiempo presente o lugar presente podrían ser sobre diferentes cosas y, en consecuencia, tener diferente contenido. La conclusión final de Stich es que aun cuando las creencias consisten en estados internos más relaciones externas, los estados internos no representarían ni se referirían a nada, y por lo tanto no serían relevantes para una creencia. Por ello, Stich afirmará que la explicación psicológica científica no hará uso de los estados que se parecen a los de la psicología popular.

\section{OBJECIONES AL ELIMINATIVISMO}

\section{Modestia de la explicación}

Baso esta réplica en la convicción compartida entre defensores y críticos del eliminativisimo, de que la psicología popular es una teoría. Como toda teoría tiene una serie de compromisos. La psicología popular, tal como es vista por el funcionalismo y la ciencia cognitiva, tiene una concepción acerca de la manera como generamos nuestra conducta a partir de un mundo mental: nuestras creencias y deseos son responsables causales de la conducta mental. Pero nótese que es una teoría modesta y no una teoría completa. Una teoría modesta acerca de lo mental no explica la naturaleza intrínseca de las entidades postuladas, las creencias y deseos. Sin embargo, dada la ubicuidad de su uso y su reconocido éxito explicativo y predictivo, las entidades postuladas parecen ser muy plausibles juzgadas por lo que conocemos de cómo se comportan las personas en diversas circunstancias.

La modestia de la teoría dentro del funcionalismo está limitada, en primer lugar, como se indicó, en que no se dice nada acerca de la naturaleza intrínseca 
de las entidades postuladas, dejando a otras disciplinas o a muchas disciplinas juntas -como en el caso de la ciencia cognitiva- la posible futura explicitación. En segundo lugar, señala los límites de su capacidad explicativa y predictiva, que es el rol que cumplen las creencias y deseos en la explicación y predicción de la conducta humana en diferentes circunstancias en el tiempo.

Los eliminativistas suelen mirar con condescendencia a la psicología popular porque la conciben como una teoría deficiente en el peor de los casos y muy incompleta en el mejor de ellos. Sin embargo, cuando tradicionalmente se ha evaluado el estatus de una teoría, se ha tomado como criterio sólo algunos aspectos involucrados. Por ejemplo, en el clásico modelo nomológico-deductivo de Hempel (1965), esperaba que toda explicación tuviera un esquema lógico rígido y que tuviera necesariamente una mención explícita de leyes. Pero pronto se advirtió que el modelo hempeliano hacía nula mención de otros elementos presentes en la explicación, como son los psicológicos y los pragmáticos.

Recordemos que las explicaciones son productos que nosotros, en forma individual o colectiva, hacemos; son el resultado de nuestra actividad en el mundo, y por lo tanto, tienen presuposiciones y consecuencias intencionales y sociales. Además, uno de los objetivos de la explicación en la comprensión de un fenómeno, y esto implica necesariamente un componente psicológico y no estrictamente lógico.

Por otro lado, la estructura y naturaleza misma de la explicación tiende a interactuar con la clase de cosa que está siendo explicada. Las interacciones causales de la biología, por ejemplo, requerirán un tipo diferente de conocimiento interpretativo que, digamos, la física. De ahí que la psicología popular puede tener lugar en el marco de diferentes formas de aproximación a los fenómenos.

Mientras que en la biología puede ser central el concepto de teleología, en la psicología la interacción causal de las creencias y deseos puede ser indispensable. Todo ello, sin que signifique que la teleología y la psicología popular agote las múltiples posibles interpretaciones que puedan surgir de los fenómenos biológicos y psicológicos, respectivamente. No se puede pretender un solo esquema explicativo en la ciencia, ni menos una "esencia explicativa". Como dicen Keil \& Wilson: "tu explicación de por qué llegaste tarde a la casa para comer y la prueba matemática de un teorema tienen poco en común" (1998, p. 140).

En otras palabras, creamos teorías para explicarnos fenómenos, pero sus presuposiciones no pueden ser, de ninguna manera, comunes, aun en una restringida economía metafísica. Por ejemplo, la intencionalidad puede ser crítica en la comprensión de los seres humanos y tendría que ser incorporada 
en una teoría acerca de su comportamiento, pero no en su comprensión.

\section{La imposibilidad de la falsificación}

P. Churchland (1991) había señalado que la psicología popular se puede volver inmune a la falsación en una forma análoga a una posible infalsabilidad del flogisto. Dice que incluso se puede resucitar la entidad postulada del flogisto. Siguiendo las líneas del funcionalismo, la teoría de la combustión del flogisto puede ser reconstruida señalando estados funcionales "altamente flogistificados" y "deflogistificados" definidos por ciertos síndromes de disposiciones causales.

Recordemos que el flogisto fue postulado para explicar el fenómeno de la combustión. Se decía que algunas sustancias eran más combustibles porque tenían más flogisto. Y la combustión misma era entendida como un proceso de deflogistificación. Hoy creemos que la combustión no tiene relación con un supuesto flogisto sino que se trata de un proceso de oxidación, y el aumento de masa se debe a la toma de oxígeno para que ocurra la combustión.

El flogisto era una noción funcionalmente asociada a la teoría del flogisto, que cumplía la siguiente regla: Que tanto más flogisto tenía una substancia cuanto más combustible era y más flogisto era emitido durante la combustión. Sin embargo, la teoría funcional del flogisto no salvó a este de la eliminación cuando se demostró que la combustión no era un proceso de emisión sino más bien de captación de algo llamado oxígeno. Lo que quiero demostrar es que, a diferencia de la teoría funcionalista de la psicología popular, el desarrollo del concepto de oxidación demostró que no había lugar para el flogisto, puesto que nada jugaba el rol de lo emitido.

El funcionalismo acerca de las creencias y deseos no parece seguir el camino del flogisto porque su rol causal sigue siendo relevante y nada parece estar en camino de desplazarlos. Parece ser que somos seres que tenemos creencias y deseos y que actuamos de acuerdo con ellos.

\section{El marco conceptual \\ de la racionalidad}

Anteriormente indiqué cuán importante puede ser en la explicación de la acción humana, el concebirnos como agentes racionales con intencionalidad. Sin embargo, en la crítica de Stich nada hace pronosticar que en el futuro la psicología científica tenga estados parecidos a las actitudes proposicionales, de tal modo que quizás ni siquiera existan estados como tales. Sin embargo, Stich considera la eliminación de las actitudes proposicionales en el contexto de la ciencia cognitiva.

La ciencia cognitiva tiene que ver con nuestras cogniciones, nuestra racionalidad. La psicología cognitiva na- 
ció del fracaso del conductismo por dar cuenta de nuestra intrincada conducta cognitiva. Algunas de nuestras conductas más complejas son mejor entendidas si suponemos que provienen de organismos que perciben, desean, detectan, reconocen, tienen metas. Eso es precisamente lo que significa ser un ser cognitivo y no un reloj. Por lo menos, quedaría a nivel de hipótesis suponer que si lo que se quiere explicar son nuestras capacidades racionales, parte de la explicación incluya las actitudes proposicionales. Pero, según Stich, la psicología cognitiva puede explicar la capacidad racional, sin apelar a estados que poseen contenido proposicional. Pero esto no es debatible.

La mejor argumentación que encuentro contra Stich está representada con el ejemplo de Pylyshyn (1980). Supongamos que una persona, digamos, Eva, viene corriendo fuera de un edificio en llamas. Si partimos de que Eva es un ser cognoscitivo, y suponemos que salir del edificio es un acto racional, tenemos que admitir que su acción tiene que haber resultado de una variedad de diferentes estímulos. Puede haber olido humo. Puede haber escuchado una alarma. Puede haber visto las llamas. Puede haber escuchado a un bombero gritar, “¡Fuera, fuego!”. De este ejemplo, y de otros, se puede comparar que, en general, todas las acciones racionales son, en lo relevante, similares. Más de un estímulo puede tener el mismo significado o importancia para el actor racional, y por lo tanto, más de un estímulo puede producir la misma acción. El acto racional es una respuesta al significado de un estímulo, pero no es el estímulo mismo.

Pero los seres cognoscitivos son seres que actúan a partir de estados internos, es decir, las causas de la acción inteligente son internas. Por ello, el conductismo era dramáticamente incompetente para dar cuenta de las acciones racionales inteligentes. Necesariamente tenemos que postular la existencia de estados internos causales o no podemos explicarnos adecuadamente el comportamiento complejo y teleológico de los humanos.

Ahora bien, para que una acción, como el escape del edificio, sea una respuesta apropiada, inteligente, a unos estímulos o a otros, el estímulo debe significar algo para el ser cognoscitivo, y este significado tiene que interactuar con los estados físicos internos para producir el comportamiento adecuado. Sin embargo, un "significado" no puede tener efecto en un organismo a menos que el significado mismo esté codificado o "realizado" de alguna manera en algún estado físico del organismo. Se puede ver que la conclusión a la que tenemos que llegar, contra Stich, es que los estados físicos de los organismos cognoscentes tienen que codificar los significados de los estímulos y tendrían que ser descritos en algún lenguaje que posea contenido proposicional. 
Los eliminativistas como Stich nos quieren conducir a la negación de la importancia de los contenidos y hacernos concluir que lo único que es importante es la referencia a estados con descripciones puramente sintácticas. Sin embargo, si uno empieza queriendo explicar el comportamiento de seres cognoscitivos, se encontrará inevitablemente incluyendo las actitudes proposicionales en las explicaciones. Revisemos por qué.

Volvamos a la escena de Eva en el incendio. Supongamos que Eva se encuentra con una serie de estímulos que puedan significar "el edificio está en llamas". Algo pasa en la cabeza de Eva y a continuación abandona corriendo el edificio. ¿Cómo podríamos reconstruir esta escena en donde vemos que su acto es una respuesta cognitiva e inteligente? Ciertamente, una descripción neurofisiológica no agotaría las capacidades racionales de ese acto particular. El hecho de que un sistema produce outputs a partir de símbolos no interpretados después de haber sido expuesto a algunos estímulos no parece explicar la conexión racional entre el acto y los estímulos. Lo que parece ser más adecuado, y aquí se puede apreciar la importancia de mantener las actitudes proposicionales, es visualizar lo que ocurre en la cabeza como símbolos que requieren interpretación y como símbolos que significan algo. Eso es precisamente lo que quieren resaltar las actitudes proposicionales.

\subsection{El factor pragmático}

Anteriormente me referí al aspecto pragmático de la explicación que fue tan descuidado en el modelo explicativo más influyente, el hempeliano. Y precisamente, uno de los elementos que más llama la atención a los defensores y detractores de la psicología popular es su notable persistencia en el tiempo y su resistencia a ser abandonada como fuente de categorías explicativas.

Los llamados "profetas del eliminativismo" (Blackburn, 1991) son de dos tipos. Los hay del tipo "Isaías" ("De la planta del pie a la cabeza, no hay en él cosa sana", Is., I: 6), y los del tipo "Juan el Bautista" (“... pero viene el que es más fuerte que yo", Lc., 3: 15). Mas pareciera que los profetas del eliminativismo seguirán esperando al Mesías, teniendo en cuenta el dato pragmático de la ubicuidad del uso de la psicología popular en las áreas profesionales de la psicología, particularmente de la psicología social.

J. Fodor hace el siguiente pronóstico:

Aun si pudiéramos prescindir de ella [la psicología popular] en principio, esto no sería ningún argumento para prescindir de ella... Lo que es relevante es si vale la pena defender su eliminación de hecho. Y aquí la situación es absolutamente clara. No tenemos ninguna idea de cómo explicarnos nosotros a nosotros mismos a excepción de un vocabulario que está saturado con psicología de la creencia y deseo. Uno está tentando a usar un argumento trascendental: lo que Kant dijo a Hume acerca de los objetos físicos, funciona mutatis mutandis para 
las actitudes proposicionales; no las podemos dejar ir porque no sabemos cómo (1985, pp. 9-10).

Fodor parece haber encontrado una de las mejores evidencias argumentativas. La psicología popular es buena porque la usamos constantemente y no se quiere ir. Por otro lado, no tenemos idea de cómo sería una explicación de la acción humana, de la vida humana subjetivamente vivida sin nuestros conceptos asociados a las actitudes proposicionales. Y, hasta el momento, a falta de contendores eficientes, nos haremos eco de las palabras de Putnam: "O aguantas o te callas" (1988, p. 79).

\section{REFERENCIAS}

Blackburn, S. (1991). Losing your mind: physics, identity and folk burglar prevention. En Greenwood, E. (Ed.). The future of folk psychology: intentionality and cognitive science. Cambridge: Cambridge University Press.

Churchland, P. (1991). Eliminative materialism and the propositional attitudes. En Rosental, D. (Ed.). The nature of mind. Nueva York: Oxford University Press.

Churchland, P. S. (1983). Consciousness: the transmutation of a concept. Pacific Philosophical Quarterly, 64, 80-95.

Churchland, P. S. (1986). Neurophilosophy. Cambridge, Mass.: MIT.
Churchland, P. \& Churchland, P. S. (1990). Intertheoretic reduction: a neuroscientist's field guide. Seminars in the neurosciences, 2, 249-256.

Feyerabend, P. (1963). Mental events and the brain. The Journal of Philosophy, 60, 295-296.

Fodor, J. (1985). Fodor's guide to mental representations. Mind, 94, 77110.

Hempel, C. (1965). Aspects of scientific explanation and other essays in the philosophy of science. Nueva York: The Free Press.

Keil, F. \& Wilson, R. A. (1998). The shadows and shallows of explanation. Minds and machines, 8, 137 159.

Kim, J. (1992). Philosophy of mind. Boulder: Westview Press.

Kim, J. (1993). Concepts of supervenience. En Kim, J. (Ed.). Supervenience and mind: selected philosophical essays (pp. 53-78). Nueva York: Cambridge University Press.

Kuhn, T. (1970). The structure of scientific revolutions. Chicago: University of Chicago Press.

Lakatos, I. (1970). Falsification and the methodology of scientific research programmes. En Lakatos, I. \& Musgrave, A. (Eds.). Criticism and the growth of knowledge. Cambridge: Cambridge University Press. 
Nagel, E. (1961). The structure of science. Nueva York: Harcourt, Brace, Jovanovich.

Place, U. T. (1956). Is Consciousness a brain process? British Journal of Pscyhology, 47, 44-50.

Putnam, H. (1988). Representation and reality. Cambridge, MA.: MIT Press.

Pylyshyn, Z. (1980). Cognitive representation and the process-architecture distinction. Behavioral and Brain Sciences, 3, 154-169.

Quine, W. V. O. (1960). Word and object. Cambridge, MA.: MIT Press.

Rorty, R. (1965). Mind-body identity, privacy and categories. Review of Metaphysics, 19, 24-54.
Schaffner, K. (1967). Approaches to reduction. Philosophy of science, 34, 137-147.

Smart, J.J.C. (1959). Sensations and brain processes. Philosophical Review, 68, 141-156.

Stich, S. (1983). From folk psychology to cognitive science. Cambridge, MA.: Bradford Books / MIT Press.

Stich, S. (1991). Autonomous psychology and the belief-desire thesis. En Rosenthal, D. (Ed.). The nature of mind. (pp. 590-612). Nueva York: Oxford University Press.

Thagard, P. (1978). Why astrology is a pseudoscience. En Hacking, I. \& Asquith, P. (Eds.). PSA 1978, vol. 1 (pp. 223-234). East Lansing: Philosophy of Science Association. 